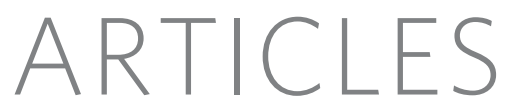

\title{
Centrosome misorientation reduces stem cell division during ageing
}

\author{
Jun Cheng ${ }^{1 *}$, Nezaket Türkel ${ }^{2 *} \uparrow$, Nahid Hemati $^{2 *}$, Margaret T. Fuller $^{4}$, Alan J. Hunt ${ }^{1} \&$ Yukiko M. Yamashita ${ }^{2,3}$
}

Asymmetric division of adult stem cells generates one self-renewing stem cell and one differentiating cell, thereby maintaining tissue homeostasis. A decline in stem cell function has been proposed to contribute to tissue ageing, although the underlying mechanism is poorly understood. Here we show that changes in the stem cell orientation with respect to the niche during ageing contribute to the decline in spermatogenesis in the male germ line of Drosophila. Throughout the cell cycle, centrosomes in germline stem cells (GSCs) are oriented within their niche and this ensures asymmetric division. We found that GSCs containing misoriented centrosomes accumulate with age and that these GSCs are arrested or delayed in the cell cycle. The cell cycle arrest is transient, and GSCs appear to re-enter the cell cycle on correction of centrosome orientation. On the basis of these findings, we propose that cell cycle arrest associated with centrosome misorientation functions as a mechanism to ensure asymmetric stem cell division, and that the inability of stem cells to maintain correct orientation during ageing contributes to the decline in spermatogenesis. We also show that some of the misoriented GSCs probably originate from dedifferentiation of spermatogonia.

Adult stem cell populations maintain highly differentiated but shortlived cells such as blood, intestinal epithelium cells and sperm throughout life. Upon division of stem cells, daughter cells must either self-renew to preserve stem cell identity or commit to differentiation. The balance between stem cell self-renewal and differentiation is critical to tissue homeostasis, with disruption of this balance leading to tumorigenesis (caused by stem cell overproliferation) or tissue degeneration (caused by stem cell depletion). To maintain this critical balance, many stem cells have the potential to divide asymmetrically, producing one daughter stem cell and one differentiating cell ${ }^{1}$. Many stem cells reside in a special microenvironment, or stem cell niche, that regulates stem cell maintenance ${ }^{2,3}$. Asymmetric stem cell division within the niche essentially relies on the correct placement of daughter cells inside and outside the niche: daughter cells that remain within the niche retain a stem cell identity whereas daughter cells displaced from the niche are fated to differentiate ${ }^{4}$. Thus, it is critical to establish stem cell polarity within the context of the niche.

A decline in the function of adult stem cells has been proposed to contribute to tissue ageing, although the underlying mechanisms remain enigmatic ${ }^{5}$. Tissue ageing has been proposed to have arisen as a tumour suppressor mechanism ${ }^{6,7}$, in which tumour suppressor activity reduces stem cell function in later stages of life, preventing tumorigenesis but reducing tissue regenerative capacity ${ }^{8}$. However, the cellular and molecular basis of such phenomena is poorly understood. Although cell cycle inhibitors such as Ink4a are known to accumulate in stem cells with age and to contribute to an age-related decline in tissue regenerative capacity ${ }^{9-11}$, the mechanisms regulating increased expression of cell cycle inhibitors and their relationship to normal stem cell function are unknown.

Male GSCs in Drosophila melanogaster always undergo asymmetric division. The divisions are regulated by a combination of signal(s) from the niche and spindle orientation. The hub cells, which constitute the stem cell niche, secrete the signalling ligand Unpaired (Upd), which activates the JAK-STAT (Janus kinase-signal transducer and activator of transcription) pathway in the neighbouring germ cells to promote stem cell maintenance ${ }^{12,13}$. The spindle lies perpendicular to the hub, so that one daughter cell inherits the attachment to the hub, while the other is displaced away from it ${ }^{14}$. This stereotypical orientation of the mitotic spindle is precisely set by the positioning of the centrosomes during interphase. The mother centrosome is always anchored to the hub-GSC interface, whereas the daughter centrosome migrates towards the opposite side of the $\mathrm{GSC}^{15}$. In this way, GSCs are oriented with respect to the niche throughout the cell cycle. Recently, similar centrosome behaviour and stem cell polarity have been reported in the Drosophila neuroblast, suggesting that centrosome orientation within stem cells has a general role in asymmetric divi$\operatorname{sion}^{16,17}$. However, the relative importance of this orientation to physiological stem cell function is unclear.

\section{Spermatogenesis declines with age in Drosophila testis}

Testes from newly eclosed males contain cells in all stages of spermatogenesis. These include transit-amplifying cells (gonialblast and 2-16-cell spermatogonia), spermatocytes, meiotic cells and elongated spermatids (Fig. 1a, b), the collective presence of which indicates ongoing spermatogenesis. In contrast, as flies age, testes undergo marked involution and the number of early germ cells in the apical region of the testis (spermatogonia, spermatocytes and meiotic cells) progressively decreases (Fig. 1b, arrow). A decrease in spermatogenesis could be attributable to decreased function of GSCs. However, the number of GSCs (defined as germ cells attached to the hub) did not significantly decrease after 30 days of age (Fig. 1c, d), when testes had already undergone significant involution. This suggests that decreased stem cell number does not fully explain the reduced spermatogenesis that is observed at this stage of ageing, although stem cell numbers ultimately decline in older flies (50 days) 

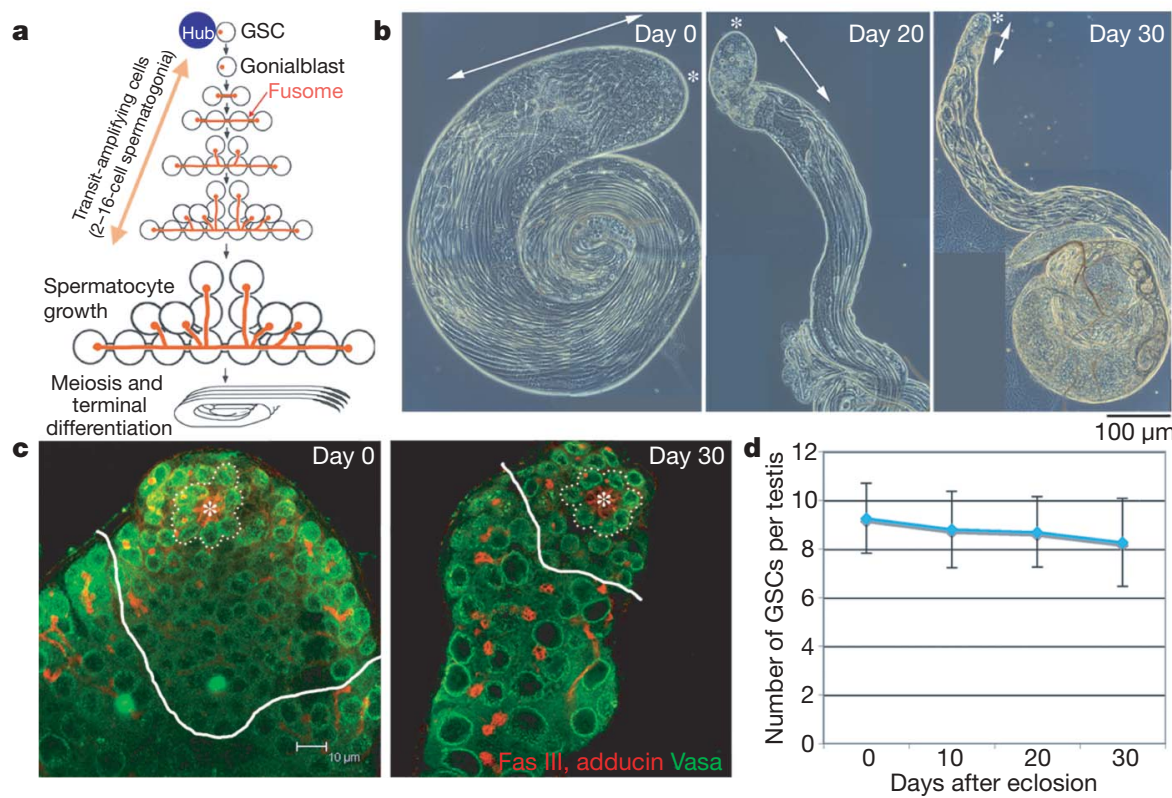

Figure 1 | Drosophila testis undergoes an age-related decline in spermatogenesis. a, Spermatogenesis of Drosophila melanogaster (adapted from ref. 27). GSCs are supported by the hub cells. Each spermatogonial division is incomplete, and the resultant spermatogonia and spermatocytes are connected by a cytoplasmic bridge, or a ring canal, through which a branched fusome runs. b, Phase microscopy of ageing testes. The apical

and may account for decreased spermatogenesis in later stages of ageing $^{18}$ (Supplementary Table 1).

\section{The ageing testis accumulates misoriented GSCs}

In Drosophila male GSCs the stereotypical orientation of the mitotic spindle is set up by the positioning of centrosomes during interphase (Fig. 2a $)^{14,15}$. In young males (0-2 days after eclosion), GSCs remain oriented towards the niche throughout the cell cycle as previously reported, setting up the stereotypically oriented mitotic spindle. However, we found that, in aged flies, GSCs in which neither centrosome was situated next to the hub became more numerous (hereafter referred to as 'misoriented centrosomes', Fig. 2b). GSCs with misoriented centrosomes increased to approximately $40 \%$ of total GSCs at 30 days after eclosion (Fig. 2c, d). It should be noted that owing to the scoring criteria (Fig. 2b), the maximum misorientation would be $\sim 50 \%$ (as each randomly positioned centrosome would end up adjacent to the hub $\sim 25 \%$ of time). Consistent with this, the frequency of misoriented GSCs reached a plateau at $\sim 40 \%$, with a similar misorientation frequency in very old flies (day 42-50, Supplementary Table 1).

\section{Misoriented GSCs divide less frequently}

Although $\sim 40 \%$ of GSCs had misoriented centrosomes in aged testes, we rarely observed 'misoriented spindles' in which neither spindle pole is associated with the hub (Fig. 2b), suggesting that GSCs with misoriented centrosomes do not enter mitosis. Overall, 95-100\% of mitotic spindles were oriented perpendicular to the hub throughout mitosis, regardless of age (Fig. 3a, b). Not only did misoriented GSCs fail to undergo mitosis, but GFP-PACT (pericentrin/ AKAP450 C-terminal domain) labelling of centrosomes revealed that significantly fewer cells with misoriented GSCs underwent the G1/S transition relative to those with oriented GSCs (Fig. 3c; see Methods). Notably, the percentage of oriented GSCs from aged flies (day 10 to 30) that entered the $S$ phase remained as high as that of oriented GSCs from young flies, demonstrating that aged GSCs do not divide less frequently if their centrosomes are appropriately oriented. In contrast, misoriented GSCs entered the S phase with a significantly lower (asterisk) area containing round, relatively early germ cells (arrow) decreases over time. c, The number of GSCs (surrounded by dotted line) remains constant with age. White lines separate spermatogonia and spermatocytes. Red, fasciclin III (Fas III; hub) and adducin (fusome); green, Vasa (germ cells). The hub is indicated by an asterisk. $d$, The number of GSCs is shown ( \pm s.d.). $n>50$ testes were counted for each time point.

a

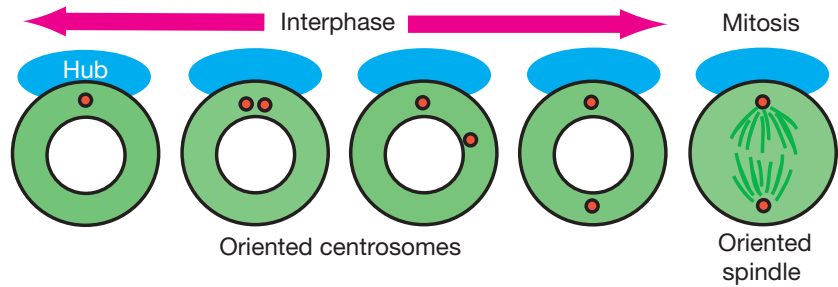

b

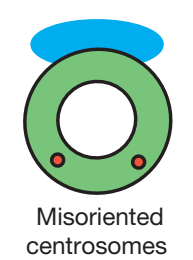

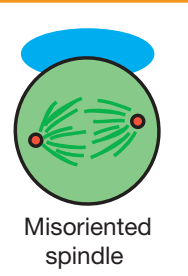
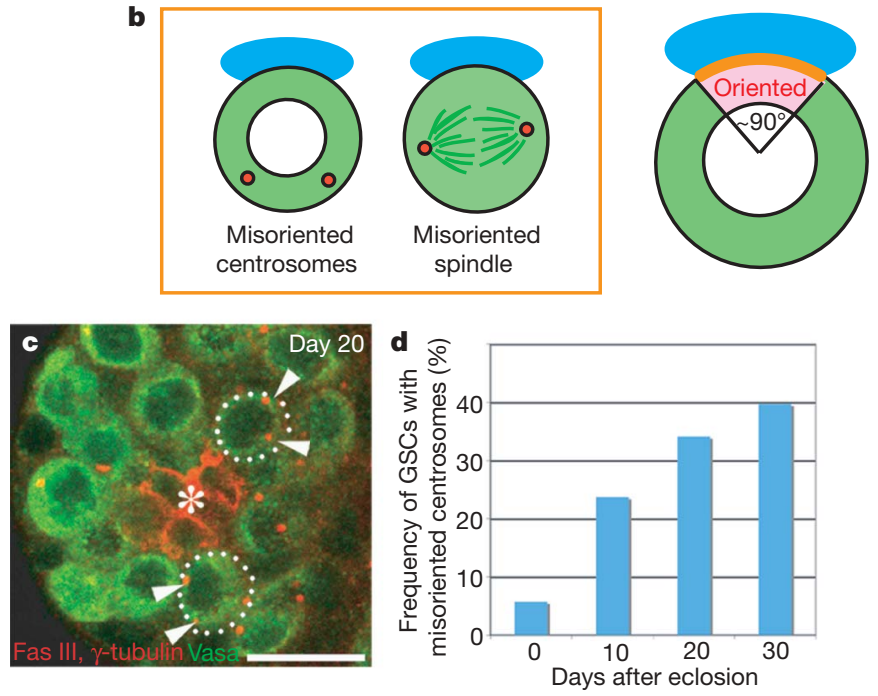

Figure 2 | Misoriented GSCs increase with age. a, Schematic diagram of centrosome positioning during the cell cycle. $\mathbf{b}$, Left panel: the definition of misoriented centrosomes and spindle. Right panel: scoring criterion.

Centrosomes were scored to be oriented when one of two centrosomes is in the pink area close to the hub-GSC junction (orange line). c, An example of testis (20-day-old) containing GSCs with misoriented centrosomes. Red, fasciclin III and $\gamma$-tubulin (centrosome); green, Vasa. The hub is indicated by an asterisk. Scale bar, $10 \mu \mathrm{m}$. d, The frequency of GSCs with misoriented centrosomes increases with age ( $n>275$ GSCs for each time point). The same trend was observed in more than three separate experiments, including conditions with different culture media and temperature $\left(22^{\circ} \mathrm{C}\right.$ to $\left.25^{\circ} \mathrm{C}\right)$. 
a

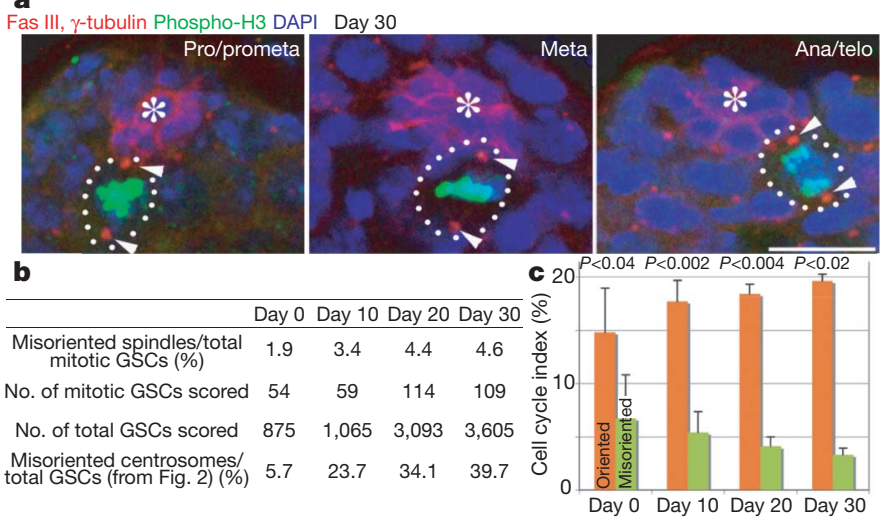

Figure 3 | Misoriented GSCs divide less frequently compared with oriented GSCs. a, Spindles remain oriented throughout mitosis even at day 30 . Red, fasciclin III and $\gamma$-tubulin; green, Thr 3-phosphorylated histone H3 (phosphoH3; mitotic chromatin); blue, DAPI. The hub is indicated by an asterisk. Scale bar, $10 \mu \mathrm{m}$. Arrowheads indicate mitotic spindle poles. $\mathbf{b}$, The frequency of misoriented spindles remained low for up to 30 days of age. The percentage of misoriented interphase cells was taken from Fig. $2 \mathrm{c}$ for comparison. c, Pulselabelling of centrosomes by heat-shock-induced GFP-PACT expression confirmed the low cell cycle activity of misoriented GSCs. The percentage of GSCs committed to the G1/S transition during the labelling period is shown (mean \pm s.d.; $n>110$ labelled GSCs for each point, equivalent to $n>750$ total GSCs per data point). $P$ values of $t$-test (two-tail) are shown.

frequency compared with oriented GSCs at all stages of life. These results suggest that the reduced division of ageing GSCs is determined by centrosome misorientation.

\section{Misoriented GSCs maintain a capability of cell division}

The rare division of misoriented GSCs raises the question of whether misorientation precedes cell cycle arrest/delay or whether it occurs as a consequence of general disintegration of stem cell activity, such as from cellular senescence. To test whether misoriented GSCs retain the ability to re-enter the cell cycle, GSCs were labelled by feeding flies with 5-bromodeoxyuridine (BrdU)-containing food from day 5 to 10 , during which time misorientation increased from approximately $10 \%$ to $20 \%$. This labelled $96 \%$ of all GSCs, including almost all of the misoriented GSCs (which accounted for $\sim 20 \%$ of all GSCs; Supplementary Fig. 1a). This demonstrates that GSCs that are misoriented by day 10 had replicated their DNA in the preceding several days. Furthermore, when BrdU was discontinued, BrdU labelling quickly disappeared during the BrdU chase period, and was completely absent after 5 days (Supplementary Fig. 1b), indicating that misoriented BrdU-positive GSCs had divided. The ability of misoriented GSCs to dilute their BrdU label indicates that misoriented GSCs are not permanently arrested in the cell cycle.

Time-lapse imaging of centrosomes in GSCs confirmed that misoriented GSCs did not divide, but were able to resume dividing as soon as centrosomes restored their correct orientation (Fig. 4). We tracked centrosome behaviour within GSCs by using mCherry-Sas6 (ref. 16), and confirmed that most GSCs exhibit stereotypical movement of centrosomes during the cell cycle as shown in Fig. 2a. In addition, we recorded 18 misoriented GSCs that underwent mitosis during the recording time. In each case, misoriented centrosomes moved around within GSCs for a long time (on average, $137 \pm 47 \mathrm{~min}$ from the start point of the recording), but as long as no centrosome aligned adjacent to the hub the cells never divided. However, when one centrosome became very close to the hub-GSC interface (or 're-oriented'), the GSCs quickly divided (in $18 \pm 12 \mathrm{~min}$ after re-orientation). The ability of GSCs to re-enter the cell cycle almost immediately on reorientation of the centrosomes suggests that centrosome misorientation is the proximal cause of the reduced GSC division and thus the testis involution observed during fly ageing.
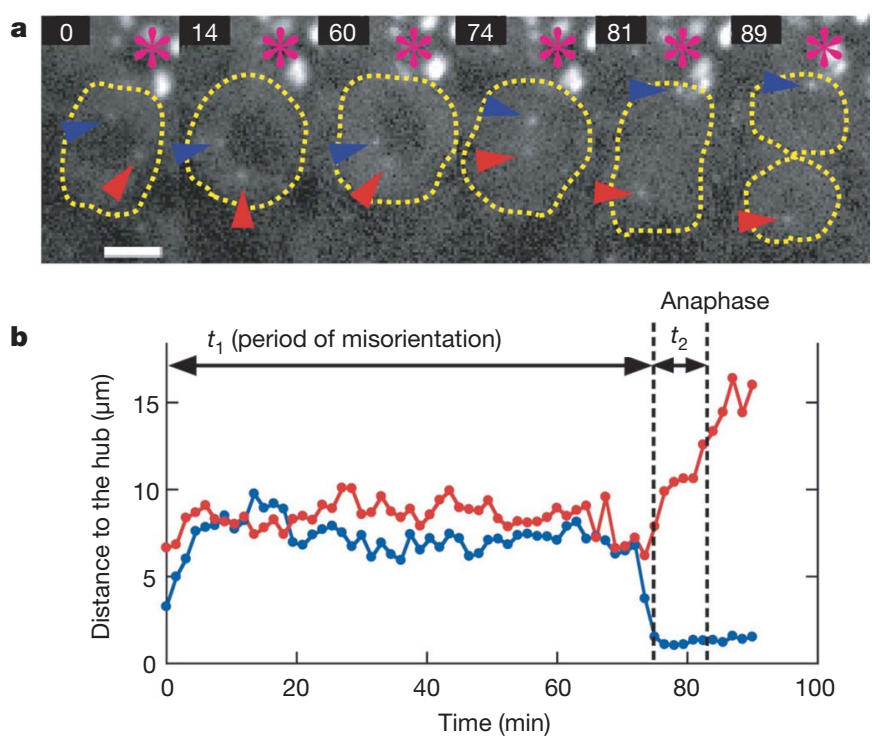

Figure 4 | Misoriented GSCs correct centrosome orientation before mitosis. a, Frames from time-lapse imaging of GSC cell division with misoriented centrosome (frames were taken from Supplementary Movie 1). Centrosome movement in a GSC was tracked by mCherry-Sas6. Numbers represent minutes from the start point of recording. Re-orientation occurred around $74 \mathrm{~min}$, and anaphase around $81 \mathrm{~min}$. The hub is indicated by an asterisk; two centrosomes are indicated by arrowheads (red and blue). Scale bar, $5 \mu \mathrm{m}$. b, Tracking of the centrosome movement from the same timelapse imaging. Red and blue lines represent the movement of centrosomes with red and blue arrowheads shown in a, respectively.

\section{Some misoriented GSCs originate from dedifferentiation}

Dedifferentiation has been proposed to be one mechanism by which the stem cell pool can be replenished ${ }^{19,20}$, although it is not clear how physiologically important this mechanism is in unperturbed tissues or how it is affected by ageing. We hypothesized that if spermatogonia dedifferentiate to stem cells, such cells might not be able to orient towards the hub initially. To test the hypothesis that misoriented GSCs may originate from dedifferentiated spermatogonia, we used FLP-recombinase-based mitotic recombination to mark permanently germ cells once committed to differentiation (Fig. 5a). FLP recombinase was expressed under the control of bam-Gal4, which is selectively expressed in differentiating cells at the 4-cell spermatogonia stage and later ${ }^{21}$. FLP activity in these cells recombined Actin promoter $>$ FRT-stop-FRT $>$ LacZ-NLS to generate a $\beta$-galactosidase expression in the differentiating cells (Actin-LacZ-NLS). In testes from third instar larvae, weak LacZ expression was apparent in 4-8-cell spermatogonia and early spermatocytes (Fig. 5b, between dashed and solid lines), whereas LacZ expression became much stronger in later spermatocytes (Fig. 5b, after solid line). This pattern of LacZ expression persisted for 50 days. Notably, we also sometimes observed LacZ expression in GSCs, as seen by LacZ staining next to the hub (Fig. 5c, arrowhead). The frequency of LacZ-positive $\left(\mathrm{LacZ}^{+}\right)$stem cells increased with age, with $0.7 \%$ GSCs being positive in third instar larvae, $6.1 \%$ in newly eclosed males and $40 \%$ in 50 day-old males (Supplementary Fig. 2). These LacZ ${ }^{+}$GSCs appear to result from dedifferentiation of spermatogonia.

To test whether the LacZ expression in GSCs might be attributable to random, spurious activation of the bam promoter in GSCs, we tested whether X-irradiation affected LacZ expression in GSCs. Because irradiation kills GSCs (as well as other cell types), this should increase the rate of dedifferentiation, whereas if LacZ expression is attributable to random bam activation in GSCs it should not be affected by irradiation. The frequency of $\mathrm{LacZ}^{+}$GSCs increased after $\mathrm{X}$-irradiation, suggesting that LacZ expression in GSCs reflects the 

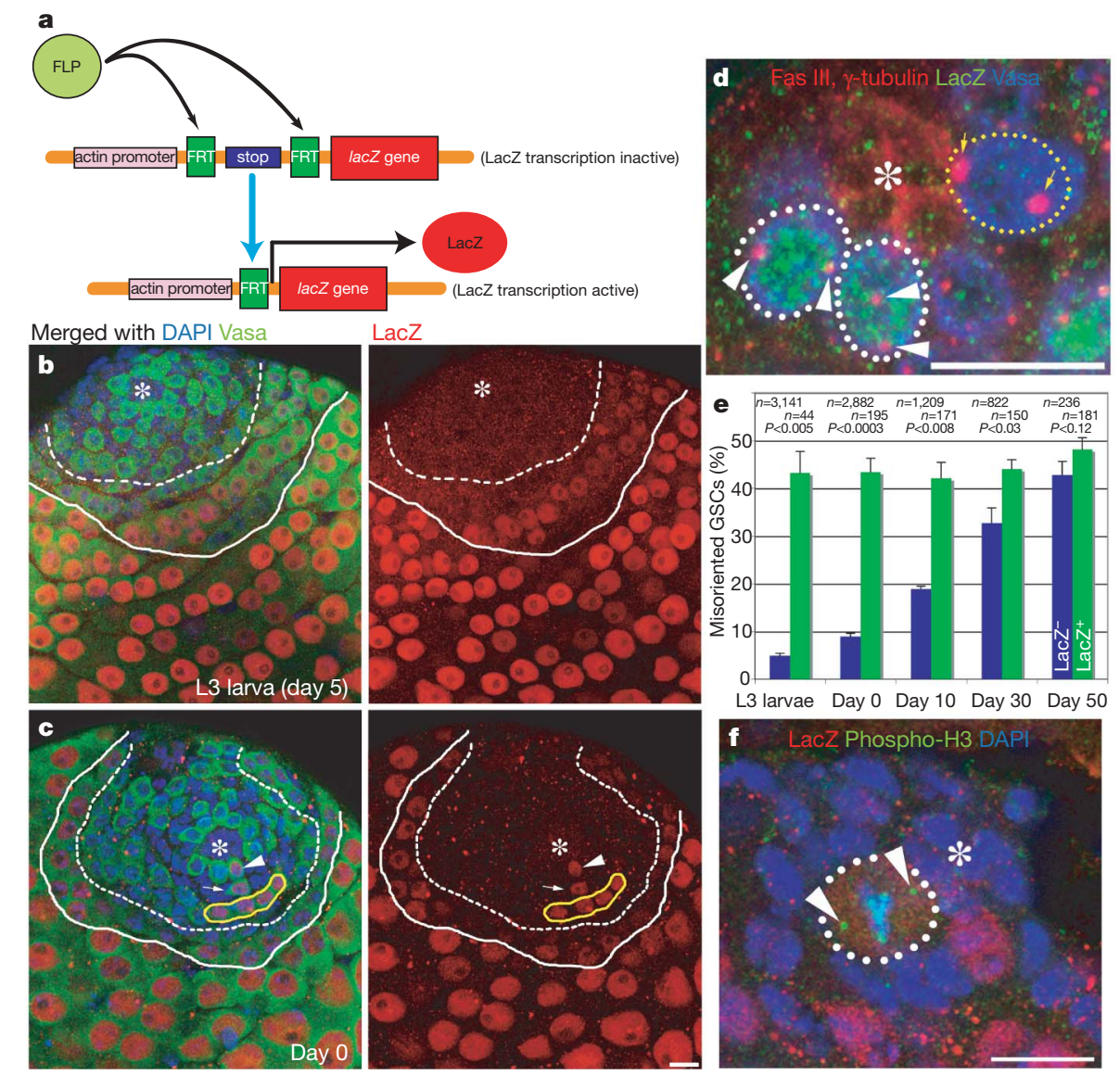

Figure 5 | Dedifferentiated GSCs have a higher frequency of centrosome misorientation. a, Strategy to label germ cells that committed to differentiation program through FLP-based recombination. b, c, LacZ staining of testes from an L3 larva (b) and a day 0 adult (c). Weak LacZ expression starts around 4-8-cell spermatogonia (dashed line) and becomes stronger in spermatocytes (solid line). The arrowhead in c shows a LacZ ${ }^{+}$ GSC with its progeny (gonialblast is shown by an arrow and 4 cell spermatogonia is surrounded by a yellow line). Red, LacZ; green, Vasa; blue, DAPI. The hub is indicated by an asterisk. d, An example of testis with two

replenishment of GSCs by dedifferentiation of spermatogonia (Fig. 6a). In further support of this conclusion, the frequency that multiple LacZ ${ }^{+}$GSCs were next to each other (Fig. 6b) was significantly higher than what would be expected based on the random, sporadic expression of FLP in GSCs (Fig. 6a), indicating simultaneous dedifferentiation of interconnected spermatogonia. Furthermore, we observed $\mathrm{LacZ}^{+}$spermatogonia next to the hub with disintegrating fusomes and ring canals (Fig. $6 \mathrm{c}$ and Supplementary Fig. 3), hallmarks of dedifferentiation ${ }^{19,20}$ (0.11 dedifferentiating events per testis after 2,000 or 3,000 rad of irradiation, $n=388$ ). Notably, we also observed many LacZ-negative ( $\mathrm{LacZ}^{-}$) dedifferentiating spermatogonia (Fig. 6d and Supplementary Fig. 3, 0.47 dedifferentiating events per testis, $n=388$ ), suggesting that not all dedifferentiation events are marked by LacZ expression (on average, only $\sim 18 \%$ of total dedifferentiation events were marked by LacZ expression). This is presumably due to the dedifferentiation from 2-4-cell spermatogonia that have not activated the bam promoter. Taken together, these results suggest that LacZ expression successfully marks dedifferentiation.

Notably, we found that $\mathrm{LacZ}^{+}$GSCs had a significantly higher frequency of centrosome misorientation compared to LacZ ${ }^{-}$GSCs (Fig. 5d, e). From L3 larvae to day 50 after eclosion, $>40 \%$ of $\mathrm{LacZ}^{+}$ GSCs showed centrosome misorientation, suggesting that dedifferentiated GSCs cannot correctly orient centrosomes towards the hub. It should be noted that not all misoriented GSCs were LacZ ${ }^{+}$. The misoriented $\mathrm{LacZ}^{+}$GSCs (white dotted line, with arrowheads indicating misoriented centrosomes) and a correctly oriented LacZ ${ }^{-}$GSC (yellow dotted line, with arrows indicating properly oriented centrosomes). Red, fasciclin III and $\gamma$-tubulin; green, LacZ; blue, Vasa. e, $\mathrm{LacZ}^{+}$GSCs have a high frequency of misorientation (mean \pm s.d.). $P$ values of $t$-test (two-tail) are shown. f, $\mathrm{LacZ}^{+}$GSCs are correctly oriented during mitosis. Fourth chromosomes that segregate before anaphase mark spindle poles (arrowheads). Red, LacZ; green, phospho-H3; blue, DAPI. Scale bars in c, d, f indicate $10 \mu \mathrm{m}$.

frequency of misoriented $\mathrm{LacZ}^{-}$GSCs increased with age, reaching $\sim 30 \%$ after 30 days and $\sim 40 \%$ after 50 days. These GSCs might result from dedifferentiation of gonialblasts and 2-4-cell spermatogonia, which have not yet expressed bam-induced FLP recombinase, as suggested above. Alternatively, there might be other reasons that cause centrosome misorientation in GSCs that remain to be determined. In either case, these results demonstrate that misoriented GSCs originate, at least in part, from the dedifferentiation of spermatogonia.

Importantly, the mitotic spindles of dedifferentiated GSCs were oriented similarly to constitutive $\left(\mathrm{LacZ}^{-}\right.$) GSCs (Fig. 5f; 97\% oriented $(n=202)$ for LacZ ${ }^{-}$GSCs and 95\% oriented $(n=20)$ for $\mathrm{LacZ}^{+}$GSCs). This demonstrates that, whereas centrosome orientation is defective in dedifferentiated GSCs, those that resume division exhibit normal mitotic spindle orientation. These data indicate that the dedifferentiated GSCs that enter mitosis do so only when their centrosomes are oriented. Considering that we rarely observed misoriented spindles, even in very early prophase (Fig. 3a), it is unlikely that the misoriented spindle (not the centrosome) undergoes reorientation after entering mitosis, as observed in Drosophila embryonic neuroblast ${ }^{22}$. This also demonstrates that dedifferentiated GSCs can function as stem cells by dividing and producing progeny, which was also demonstrated by the presence of $\mathrm{Lac}^{+}$differentiating progenies produced by LacZ ${ }^{+}$GSCs (Fig. 5c; arrow, gonialblast; yellow solid line, 4-cell spermatogonia). 


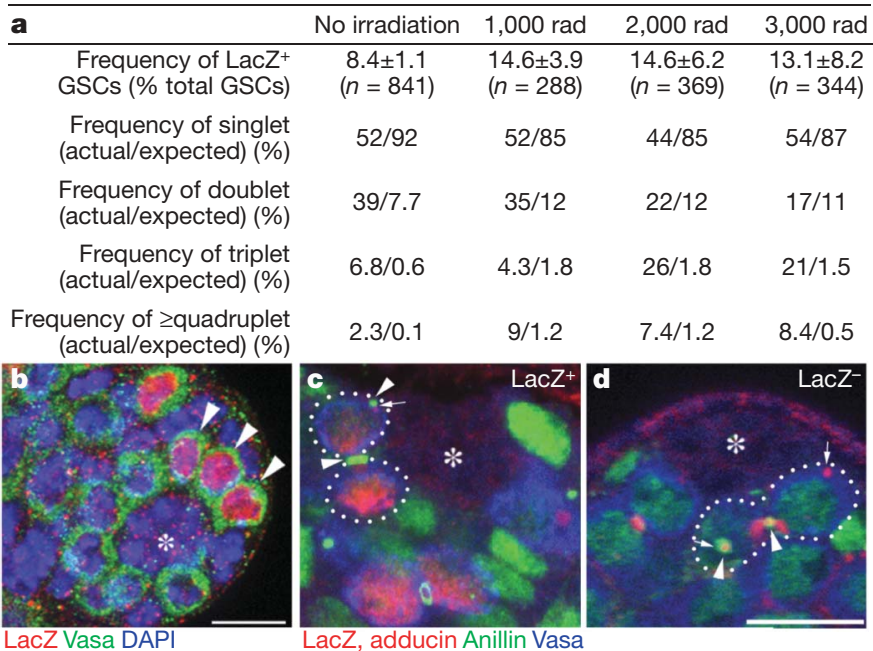

Figure 6 | Evidence that LacZ ${ }^{+}$GSCs are generated by

dedifferentiation a, Summary of $\mathrm{LacZ}^{+}$GSC frequencies with or without $\mathrm{X}$-ray irradiation. Flies were irradiated at day 0 of age, and analysed at day 5 . The actual and expected frequencies of singlet, doublet, triplet and quadruplet (or more) are shown. $n$ indicates the number of GSCs scored. The expected frequencies were calculated as described in Methods. $\mathbf{b}$, An example of testis apical tip (irradiated with 2,000 rad) containing a triplet (arrowheads: three $\mathrm{LacZ}^{+}$GSCs are locating next each other). Red, LacZ; green, Vasa; blue, DAPI. Scale bar, $10 \mu \mathrm{m}$. The hub is indicated by an asterisk. c, d, Examples of $\mathrm{LacZ}^{+}$(c) and $\mathrm{LacZ}^{-}$(d) dedifferentiating spermatogonia (surrounded by dotted lines), with multiple ring canals (arrowheads) and disintegrating fusomes (arrows), observed $24 \mathrm{~h}$ after irradiation. Red, LacZ and adducin; green, anillin (ring canal and nucleus); blue, Vasa. Scale bar, $10 \mu \mathrm{m}$.

\section{Discussion}

Here we demonstrate that GSCs with misoriented centrosomes accumulate as flies age. Because such misoriented GSCs divide less frequently as compared with oriented GSCs, accumulation of misoriented GSCs contributes to the decline in spermatogenesis that occurs with age. Although misoriented GSCs rarely divide, they are not permanently arrested (or senescent) and are correctly oriented when they divide. Whether correction of GSC orientation is an active process that is part of the acquisition of stem cell identity remains to be determined. The low cell cycle activity of misoriented GSCs may also suggest that mechanisms are in place to detect misorientation and induce cell cycle arrest in response to this change, although the underlying mechanisms remain to be identified.

We have also demonstrated that misoriented GSCs originate, at least in part, from dedifferentiation of spermatogonia. Although dedifferentiated GSCs have a high frequency $(>40 \%)$ of centrosome misorientation they can function as stem cells by resuming the cell cycle, with correctly oriented mitotic spindles just like constitutive GSCs. GSC numbers do not decrease as quickly as expected from the calculated GSC half-life, suggesting that a mechanism to compensate for the loss of GSCs exists ${ }^{23}$. Because we rarely observed misoriented spindles, or symmetric stem cell division, we speculate that dedifferentiation is the major mechanism to replace stem cells over time in the Drosophila male germ line.

A decline in GSC number in older males (day 50) was reported recently ${ }^{18}$. This decrease in stem cell number is probably due to failure of the niche function (via decreased signal from the niche as well as decreased E-cadherin-based attachment between the niche and GSCs) ${ }^{18}$. However, the decrease in the production of spermatogonia and testis involution precede the loss of GSCs such that decreasing GSC numbers cannot explain the testis involution that is observed at younger ages.

The present results provide a novel mechanistic link between the control of stem cell polarity and the age-related decline in tissue regenerative capacity. Mechanisms responsible for monitoring stem cell orientation with respect to the niche not only prevent overproliferation of stem cells by ensuring the asymmetric outcome of the stem cell division, but they contribute to the decline in tissue regenerative capacity during ageing. Many of the misoriented GSCs originate from the dedifferentiation of spermatogonia, a mechanism thought to be responsible for maintaining the stem cell population over extended periods of time. Therefore, although GSCs produce fewer progeny over time, the system appears to maximize the number of progeny produced throughout life, while maintaining asymmetric stem cell division.

We propose that the GSCs with misoriented centrosomes divide less frequently and that a combination of such a decreased stem cell division and a higher frequency of the GSC misorientation in aged testes leads to a decline in spermatogenesis with age.

\section{METHODS SUMMARY}

Immunofluorescent staining. Immunofluorescent staining was performed as described elsewhere ${ }^{14}$. For $\gamma$-tubulin and LacZ dual staining, testes were fixed in $90 \%$ ethanol $3.8 \%$ formaldehyde solution (chilled to $-20^{\circ} \mathrm{C}$ ). Fixed testes were then permeabilized by washing in $1 \times$ PBS with $0.1 \%$ Triton X-100 (30 min) before immunofluorescent staining. The following primary antibodies were used in combination with appropriate Alexa-Fluor-conjugated secondary antibodies (1:200, Molecular Probes): mouse anti-fasciclin III (1:10, developed in ref. 24 and obtained from the Developmental Studies Hybridoma Bank), mouse antiadducin (1:20, developed in ref. 25 and obtained from the Developmental Studies Hybridoma Bank), rabbit anti-Vasa (1:2,000, a gift from R. Lehmann), goat anti-Vasa (1:20, Santa Cruz), mouse anti- $\gamma$-tubulin $(1: 100$, GTU-88, Sigma), rabbit anti-phosphorylated histone H3 (Thr3) (1:200, Upstate), mouse anti- $\beta$-galactosidase (1:200, G4644, Sigma), rabbit anti- $\beta$-galactosidase (1:500, Abcam), rabbit anti-anillin (1:1,300, a gift from C. Field) and mouse anti-BrdU (1:200, BU-33, Sigma). BrdU staining was performed as detailed in ref. 26. Images were taken using a Leica SP5 confocal microscope and processed using Adobe Photoshop CS 8.0.

Time-lapse live imaging of GSCs inside testes. Newly eclosed Sas6-mCherry flies ${ }^{16}$ were dissected inside Drosophila culture medium containing Schneider's Drosophila medium and $10 \%$ fetal bovine serum. The testis tips were placed inside a sterile glass-bottom chamber, and was mounted on a three-axis computer-controlled piezoelectric stage and imaged using an inverted microscope equipped with an electron multiplier cooled CCD camera. Four-dimensional image sequences ( $x, y, z$ and time) were acquired every $90 \mathrm{~s}$. A semi-automatic tracking software was used to track locations of centrosomes and hubs, and the tracking algorithm was based on the pattern matching routine inside National Instrument IMAQ Vision software. The Supplementary Movie was generated using Sony Vegas software.

Full Methods and any associated references are available in the online version of the paper at www.nature.com/nature.

Received 1 April; accepted 1 September 2008.

Published online 15 October 2008.

1. Morrison, S. J. \& Kimble, J. Asymmetric and symmetric stem-cell divisions in development and cancer. Nature 441, 1068-1074 (2006).

2. Spradling, A., Drummond-Barbosa, D. \& Kai, T. Stem cells find their niche. Nature 414, 98-104 (2001).

3. Fuchs, E., Tumbar, T. \& Guasch, G. Socializing with the neighbors: stem cells and their niche. Cell 116, 769-778 (2004)

4. Yamashita, Y. M., Fuller, M. T. \& Jones, D. L. Signaling in stem cell niches: lessons from the Drosophila germline. J. Cell Sci. 118, 665-672 (2005).

5. Rando, T. A. Stem cells, ageing and the quest for immortality. Nature 441, 1080-1086 (2006).

6. Sharpless, N. E. \& Depinho, R. A. How stem cells age and why this makes us grow old. Nature Rev. Mol. Cell Biol. 8, 703-713 (2007).

7. Serrano, M. \& Blasco, M. A. Cancer and ageing: convergent and divergent mechanisms. Nature Rev. Mol. Cell Biol. 8, 715-722 (2007).

8. Kirkwood, T. B. Understanding the odd science of aging. Cell 120, 437-447 (2005).

9. Krishnamurthy, J. et al. p16 ${ }^{\text {INK4a }}$ induces an age-dependent decline in islet regenerative potential. Nature 443, 453-457 (2006).

10. Molofsky, A. V. et al. Increasing p16 $16^{\text {INK } 4 a}$ expression decreases forebrain progenitors and neurogenesis during ageing. Nature 443, 448-452 (2006)

11. Janzen, V. et al. Stem-cell ageing modified by the cyclin-dependent kinase inhibitor p16 ${ }^{\text {INK4a }}$. Nature 443, 421-426 (2006).

12. Tulina, N. \& Matunis, E. Control of stem cell self-renewal in Drosophila spermatogenesis by JAK-STAT signaling. Science 294, 2546-2549 (2001). 
13. Kiger, A. A., Jones, D. L., Schulz, C., Rogers, M. B. \& Fuller, M. T. Stem cell selfrenewal specified by JAK-STAT activation in response to a support cell cue. Science 294, 2542-2545 (2001).

14. Yamashita, Y. M., Jones, D. L. \& Fuller, M. T. Orientation of asymmetric stem cell division by the APC tumor suppressor and centrosome. Science 301, 1547-1550 (2003).

15. Yamashita, Y. M., Mahowald, A. P., Perlin, J. R. \& Fuller, M. T. Asymmetric inheritance of mother versus daughter centrosome in stem cell division. Science 315, 518-521 (2007)

16. Rusan, N. M. \& Peifer, M. A role for a novel centrosome cycle in asymmetric cell division. J. Cell Biol. 177, 13-20 (2007).

17. Rebollo, E. et al. Functionally unequal centrosomes drive spindle orientation in asymmetrically dividing Drosophila neural stem cells. Dev. Cell 12, 467-474 (2007).

18. Boyle, M., Wong, C., Rocha, M. \& Jones, D. L. Decline in self-renewal factors contributes to aging of the stem cell niche in the Drosophila testis. Cell Stem Cell 1 , 470-478 (2007).

19. Brawley, C. \& Matunis, E. Regeneration of male germline stem cells by spermatogonial dedifferentiation in vivo. Science 304, 1331-1334 (2004).

20. Kai, T. \& Spradling, A. Differentiating germ cells can revert into functional stem cells in Drosophila melanogaster ovaries. Nature 428, 564-569 (2004).

21. Schulz, C. et al. A misexpression screen reveals effects of bag-of-marbles and TGF $\beta$ class signaling on the Drosophila male germ-line stem cell lineage. Genetics 167, 707-723 (2004)

22. Kaltschmidt, J. A., Davidson, C. M., Brown, N. H. \& Brand, A. H. Rotation and asymmetry of the mitotic spindle direct asymmetric cell division in the developing central nervous system. Nature Cell Biol. 2, 7-12 (2000).

23. Wallenfang, M. R., Nayak, R. \& DiNardo, S. Dynamics of the male germline stem cell population during aging of Drosophila melanogaster. Aging Cell 5, 297-304 (2006)
24. Patel, N. H., Snow, P. M. \& Goodman, C. S. Characterization and cloning of fasciclin III: a glycoprotein expressed on a subset of neurons and axon pathways in Drosophila. Cell 48, 975-988 (1987).

25. Ding, D., Parkhurst, S. M. \& Lipshitz, H. D. Different genetic requirements for anterior RNA localization revealed by the distribution of Adducin-like transcripts during Drosophila oogenesis. Proc. Natl Acad. Sci. USA 90, 2512-2516 (1993).

26. Gonczy, P. \& DiNardo, S. The germ line regulates somatic cyst cell proliferation and fate during Drosophila spermatogenesis. Development 122, 2437-2447 (1996).

27. Fuller, M. T. Spermatogenesis. In The Development of Drosophila melanogaste (eds Bate, M. \& Martinez-Arias, A.) 71-147 (Cold Spring Harbor Laboratory Press, 1993).

Supplementary Information is linked to the online version of the paper at www.nature.com/nature.

Acknowledgements We thank C. Gonzalez, D. McKearin, N. Rusan, M. Peifer and the Bloomington Stock Center for fly stocks; R. Lehmann, C. Field and the Developmental Studies Hybridoma Bank for antibodies; M. Kiel and D. Nakada for help with X-ray irradiation; and S. Morrison and T. Mahowald for comments on the manuscript. This research was supported by a University of Michigan start-up fund, March of Dimes Basil O'Conner Starter Scholar Research Award and the Searle Scholar Program (to Y.M.Y.), and NIH grants P01 DK53074 (to M.T.F.) and R01GM072006 (to A.J.H.)

Author Contributions Y.M.Y. designed research. J.C. and A.J.H. designed and conducted time-lapse imaging of centrosome behaviour. J.C., N.T., N.H. and Y.M.Y. performed other experiments. M.T.F. contributed to research design for Figs 1 and 2. Y.M.Y. wrote the manuscript.

Author Information Reprints and permissions information is available at www.nature.com/reprints. Correspondence and requests for materials should be addressed to Y.M.Y. (yukikomy@umich.edu). 


\section{METHODS}

Fly strains. Heat-shock (hs)-Gal4 (Flybase), Actin $<$ FRT-stop-FRT $<$ LacZ and UAS-FLP flies were obtained from the Bloomington Drosophila Stock Center. UAS-GFP-PACT has been described previously ${ }^{15}$. bam-Gal4 is a gift from D. McKearin. mCherry-Sas6 is a gift from N. Rusan and M. Peifer ${ }^{16}$. All Drosophila stocks were maintained under standard culture conditions using Bloomington Standard media or yeast-glucose media.

Cell cycle index assessed by GFP-PACT incorporation into centrosome. Heatshock (hs)-Gal4/UAS-GFP-PACT flies ( $0,10,20,30$ days of age) were subjected to $2.5 \mathrm{~h}$ of heat shock at $37^{\circ} \mathrm{C}$. Cells that passed through the G1/S transition during the period of GFP-PACT expression incorporated GFP-PACT into their centrosomes. At $10 \mathrm{~h}$ after the heat shock, when most of the GSCs are still in the first cell cycle, flies were dissected and testes were subjected to immunofluorescent staining. Cell cycle index was calculated as GFP-positive GSCs/total GSCs (\%).

Pulse-chase of BrdU labelling. $y w$ or Asl-YFP flies (a gift from C. Gonzalez) were fed from 6 days of age to 10 days of age with BrdU in the mixture of $1 \mathrm{ml}$ $100 \%$ apple juice, $0.8 \mathrm{~g}$ of instant fly food (Sigma) and $40 \mu \mathrm{lof} 100 \mathrm{mg} \mathrm{ml}^{-1}$ BrdU solution (in 1:1 of acetone and DMSO). Flies were transferred to fresh BrdU-containing food every $24 \mathrm{~h}$. After the feeding period, flies were transferred to regular fly food and subjected to immunofluorescent staining at appropriate time points (day 10 (0-day chase), day 12 (2-day chase) and day 15 (5-day chase)). Some flies did not incorporate BrdU into gonad; thus such testes without any BrdU incorporation were not scored.

Detection of germ cells that have committed to the differentiation program. Male UAS-FLP; bam-Gal4 flies were crossed to female Actin<FRT-stopFRT $<$ LacZ-NLS flies. Progenies were aged appropriately and subjected to immunofluorescent staining for LacZ and other appropriate markers. For X-ray irradiation, flies were irradiated with a caesium 137 GammaCell40 Exactor Irradiator (MDS Nordia), delivering approximately $100 \mathrm{rad} \mathrm{min}^{-1}$.

It should be noted that we occasionally observed $\mathrm{LacZ}^{+}$signal in testicular somatic cells (that is, cyst progenitor cells, cyst cells and hub cells), although much less frequently than $\mathrm{LacZ}^{+}$GSCs. In contrast to $\mathrm{LacZ}^{+}$GSCs, of which frequency increases with age, the frequency of the $\mathrm{LacZ}^{+}$somatic cells did not increase over time (L3 larvae to 30 days of age), suggesting that somatic cells from late larval stage to 30 days of age do not newly create active lacZ gene. Although we do not know the reason why lac Z gene activation occurs in somatic cells, such events appear to occur only during early stages of development but not after the L3 larval stage.

Statistical analysis of $\mathrm{LacZ}^{+}$GSC frequency. With or without X-irradiation, the frequency of $\mathrm{LacZ}^{+} \mathrm{GSCs}(x)$ comes from actual observation and is calculated as the number of counted $\mathrm{LacZ}^{+}$GSCs divided by the total number of observed GSCs. Then, the frequency of $\mathrm{LacZ}^{-}$GSCs is $1-x$. A general statistical analysis is discussed below, excluding unrealistic cases of $x=0$ or 1 (that is, all GSCs are $\mathrm{LacZ}^{-}$or $\mathrm{LacZ}^{+}$).

The singlet is defined as a single $\mathrm{LacZ}^{+}$GSC with adjacent $\mathrm{LacZ}^{-}$GSCs on both sides, and the doublet is defined as two $\mathrm{LacZ}^{+}$GSCs next to each other with adjacent LacZ ${ }^{-}$GSCs on both sides, and so on. In Fig. 6, the expected frequency was calculated as the 'conditional probability' with the assumption of random LacZ expression in GSCs. Because $x \neq 0$ or 1 , the probability of locating an alignment of $\mathrm{LacZ}^{+}$next to $\mathrm{LacZ}^{-}$GSC (that is, $\left[\mathrm{LacZ}^{-}, \mathrm{LacZ}^{+}\right]$) is unity. Under this condition, if the next GSC is $\mathrm{LacZ}^{-}$(with the probability $1-x$ ), it is counted as a singlet (that is, $\left[\mathrm{LacZ}^{-}, \mathrm{LacZ}^{+}, \mathrm{LacZ}^{-}\right]$). Thus, the probability of singlet is $1-x$. If an alignment of $\left[\mathrm{LacZ}^{-}, \mathrm{LacZ}^{+}\right]$is followed by a $\mathrm{LacZ}^{+} \mathrm{GSC}$ (with the probability $x$ ) and then a LacZ ${ }^{-}$GSC (with the probability $1-x$ ), it is counted as a doublet (that is, $\left[\mathrm{LacZ}^{-}, \mathrm{LacZ}^{+}, \mathrm{LacZ}^{+}, \mathrm{LacZ}^{-}\right]$), resulting in the probability of doublet as $(1-x) x$. Similarly, the probability of triplet (that is, $\left.\left[\mathrm{LacZ}^{-}, \mathrm{LacZ}^{+}, \mathrm{LacZ}^{+}, \mathrm{LacZ}^{+}, \mathrm{LacZ}^{-}\right]\right)$is $(1-x) x^{2}$, and the probability of quadruplet or more is $(1-x) x^{3}+(1-x) x^{4}+\cdots=x^{3}$. Here, we did not consider the fact that GSCs are aligned in a circle around the hub, because the probability of all GSCs surrounding the hub ( $\sim 9$ GSCs per testis) being $\mathrm{LacZ}^{+}$is negligible for either actual observations or calculated expected values $\left(<10^{-7}\right)$. Obviously, the normalization of the total probability is also conserved by summing all probability together as $(1-x)+(1-x) x+(1-x) x^{2}+x^{3}=1$. 